\title{
Potencial Evocado Auditivo Mismatch Negativity em músicos e não músicos: Revisão
}

\section{sistematica}

\author{
Auditive Mismatch Negativity Evoked Potential in musicians and non - musicians: Systematic \\ review
}

Posible Desajuste Auditivo Evocado de Negatividad en músicos y no músicos: Revisión sistemática

Recebido: 19/01/2021 | Revisado: 23/01/2021 | Aceito: 25/01/2021 | Publicado: 31/01/2021

\author{
Ainoã Ruthy Farias Silva Veras \\ ORCID: https://orcid.org/0000-0003-3820-0406 \\ Faculdade de Ensino Superior do Piauí, Brasil \\ E-mail: ainoaveras2@gmail.com \\ Ysa Karen Santos Macambira \\ ORCID: https://orcid.org/0000-0001-7061-7880 \\ Faculdade de Ensino Superior do Piauí, Brasil \\ E-mail: ysakaren@gmail.com \\ Ruth Raquel Soares de Farias \\ ORCID: https://orcid.org/0000-0002-0988-0900 \\ Faculdade do Ensino Superior do Piauí, Brasil \\ E-mail: ruthraquelsf@gmail.com
}

\begin{abstract}
Resumo
O potencial evocado de longa latência Mismatch Negativity (MMN) se refere de forma objetiva como método eletrofisiológico às habilidades de discriminação sonora, processamento auditivo, memória auditiva e atenção involuntária em nível central. Tais habilidades são encontradas de maneira distinta entre músicos e não músicos e o MMN é utilizado de forma objetiva para detecta-las. Objetivo: realizar uma revisão sistemática para verificar se existe diferenças de latência e amplitude apresentadas por meio do MMN em músicos e não músicos. Método: A pesquisa foi realizada entre setembro e novembro de 2020, nas bases eletrônicas Pubmed, ScienceDirect, BVS (Lilacs), Scopus, Circumpolar Health Bibliographic Database, SciELO, Embase, Web of science e Cochrane Central Register of Controlled Trials (CENTRAL), bem como as bases de literatura cinzenta OpenGrey.eu, DissOnline, The New York Academy of Medicine, Clinical Evidence e Clinical Trials, utilizando os descritores da seguinte forma Music OR musician OR musicians AND potencial mismatch negavitity OR MMN OR evoked potentials auditory OR Long Latency. Resultados: Foram encontrados 9 artigos, sendo que 7 abordavam que os músicos superavam os não músicos em suas latências e amplitudes de $\mathrm{MMN}$, em contraste 2 artigos não diferenciavam as latências e amplitudes entre músicos e não músicos. Conclusão: Os músicos apresentaram em uma grande maioria dos estudos uma superioridade aos não músicos em amplitude, o que significa que as habilidades neurais de discriminação auditiva e comportamentais dos músicos superam aos de não músicos, os músicos apresentaram menor latência de MMN ainda assim superando os não músicos.
\end{abstract}

Palavras-chave: Potencial evocado auditivo; Músicos; Não músicos; MMN.

\begin{abstract}
The evoked potential of long latency Mismatch Negativity (MMN) refers objectively as an electrophysiological method to the abilities of sound discrimination, auditory processing, auditory memory and involuntary attention at central level. Such skills are found differently between musicians and non-musicians and MMN is used in an objective way to detect them. Objective: to carry out a systematic review to check if there are differences in latency and amplitude presented by MMN in musicians and non-musicians. Method: The research was carried out between September and November 2020, in the electronic databases Pubmed, ScienceDirect, BVS (Lilacs), Scopus, Circumpolar Health Bibliographic Database, SciELO, Embase, Web of science and Cochrane Central Register of Controlled Trials (CENTRAL), as well as the gray literature bases OpenGrey.eu, DissOnline, The New York Academy of Medicine, Clinical Evidence and Clinical Trials, using the descriptors as follows Music OR musician OR musicians AND potential mismatch negavitity OR MMN OR evoked potentials auditory OR Long Latency . Results: 9 articles were found, of which 7 addressed that musicians outperformed non-musicians in their latencies and amplitudes of MMN, in contrast 2 articles did not differentiate the latencies and amplitudes between musicians and non-musicians. Conclusion: In a large majority of studies, musicians showed a superiority to non-musicians in amplitude, which means that the neural abilities of auditory and behavioral discrimination of musicians surpass those of non-musicians, musicians presented lower latency of MMN, even exceeding not musicians.
\end{abstract}

Keywords: Auditory evoked potential; Musicians; No musicians; MMN. 


\section{Resumen}

El potencial evocado de la negatividad de desajuste de latencia prolongada (MMN) se refiere objetivamente como método electrofisiológico a las capacidades de discriminación de sonido, procesamiento auditivo, memoria auditiva y atención involuntaria a nivel central. Tales habilidades se encuentran de manera diferente entre músicos y no músicos y MMN se utiliza de manera objetiva para detectarlas. Objetivo: realizar una revisión sistemática para comprobar si existen diferencias en la latencia y amplitud que presenta MMN en músicos y no músicos. Método: La investigación se realizó entre septiembre y noviembre de 2020, en las bases de datos electrónicas Pubmed, ScienceDirect, BVS (Lilacs), Scopus, Circumpolar Health Bibliographic Database, SciELO, Embase, Web of science y Cochrane Central Register of Controlled Trials (CENTRAL) así como las bases de la literatura gris OpenGrey.eu, DissOnline, The New York Academy of Medicine, Clinical Evidence y Clinical Trials, utilizando los descriptores de la siguiente manera Música O músico O músicos Y posible negavitidad de desajuste O MMN O potenciales evocados auditivos O Larga latencia . Resultados: se encontraron 9 artículos, de los cuales 7 abordaron que los músicos superaron a los no músicos en sus latencias y amplitudes de $\mathrm{MMN}$, en contraste 2 artículos no diferenciaron las latencias y amplitudes entre músicos y no músicos. Conclusión: En una gran mayoría de estudios, los músicos mostraron una superioridad sobre los no músicos en amplitud, lo que significa que las habilidades neuronales de discriminación auditiva y conductual de los músicos superan a las de los no músicos, los músicos presentaron menor latencia de MMN, incluso superando no músicos.

Palabras clave: Potenciales evocados auditivos; Músicos; No músicos; MMN.

\section{Introduçãa}

O Mismatch Negativity (MMN) é um potencial evocado auditivo de longa latência, gerado por meio de respostas eletrofisiológicas que se referem às habilidades de discriminação sonora, processamento auditivo, memória auditiva e atenção involuntária em nível central (Ferreira, Bueno, Costa, \& Sleifer, 2017). Tais habilidades cognitivas, características estruturais e encefálicas tem sido alvo de estudos sobre os processos neurais auditivos em pessoas com habilidades auditivas excepcionais, como os músicos, que são expostos à percepção e o processamento da música (Nikjeh, Lister, \& Frisch, 2009).

O treinamento musical, que geralmente acontece nos primeiros anos de vida, pode levar o cérebro a gerar adaptações estruturais, como mudanças nas conexões sinápticas e organização plástica. Assim, é determinante levar em consideração o tempo de treino dos músicos, já as experiências consolidam aspectos de aprendizagem na memória auditiva (Rodrigues, Loureiro, \& Caramelli, 2013). Diferenças funcionais encontradas entre os cérebros dos músicos e não músicos são detectadas pelos mismatch negativity (MMN), os resultados encontrados sugerem que músicos têm aspectos de memória sensorial auditiva pré-atenta superiores aos não músicos, isso sugere que os músicos detectam diferenças auditivas indiscrimináveis pelo não músicos em nível pré- atencioso, o que nos diz que a experiencia musical pode ser aprimorada por traços de memória sensorial que contém informações auditivas, além disso músicos discriminam e respondem modificações de pitch de forma mais rápidas apresentando latências curtas de MMN a intervalos padrões familiares, não familiares, e desvios de tons harmônicos complexos, já suas amplitudes são maiores em relação as mudanças de tom (Nikjeh et al., 2009).

O MMN avalia em nível de córtex auditivo, e suas respostas são obtidas por meio de estímulos idênticos (padrão) e estímulos acusticamente diferentes (desviantes). Os estímulos idênticos são apresentados em blocos, que são substituídos aleatoriamente pelos estímulos desviantes. As diferenças encontradas são referentes à frequência, intensidade, duração, caraterísticas fonéticas, localização espacial, rítmica, amplitude, latência e padrão temporal (Rodrigues et al., 2013).

Segundo (Ferreira et al., 2017), o Mismatch Negativity de forma espontânea demonstra seus resultados em formato de onda com polaridade negativa (negativity), não necessitando da atenção do indivíduo aos estímulos sonoros. A obtenção dos resultados acontece ao subtrair a detecção de sons inesperados (mistmatch), e sons já armazenados na memória sensorial auditiva de curto prazo. Assim, a análise das latências e amplitude de onda do MMN são importantes, já que a latência indica o tempo de processamento da atividade, enquanto a extensão da alocação neural é dada pela amplitude de onda envolvida nos processos cognitivos dos potencias.

Desta forma o MMN auxilia nos resultados no que se refere à percepção, discriminação sonora, processamento auditivo, atenção e memória, demonstrando alterações ou não no processamento auditivo. Com isto este estudo teve como 
objetivo realizar uma revisão sistemática para verificar se existe diferenças de latência e amplitude apresentadas por meio do MMN em músicos e não músicos.

\section{Metodologia}

O estudo trata-se de uma revisão sistemática, com abordagem de natureza quantitativa, os dados foram coletados e analisados (Pereira, Shitsuka, Parreira, \& Shitsuka, 2018). Tendo como objetivo responder a seguinte questão: "Existe diferença entre as latências e amplitudes de MMN entre músicos e não músicos?”, esta revisão sistemática foi realizada entre setembro e novembro de 2020, nas bases eletrônicas Pubmed, ScienceDirect, BVS (Lilacs), Scopus, Circumpolar Health Bibliographic Database, SciELO, Embase, Web of science e Cochrane Central Register of Controlled Trials (CENTRAL), bem como as bases de literatura cinzenta OpenGrey.eu, DissOnline, The New York Academy of Medicine, Clinical Evidence e Clinical Trials. Houve seleção dos descritores e termos livres, tendo como site de busca Medical Subject Headings (MeSH) para os descritores pesquisados em inglês.

Como estratégia de busca teve como base os elementos do PICO, os descritores foram dispostos da seguinte forma: (Music OR musician OR musicians) AND (potencial mismatch negavitity OR MMN OR evoked potentials auditory OR Long Latency). Buscou-se estudos realizados por meio MMN em músicos e não músicos, os termos "mismatch" e "negativity", não foram encontrados no Medical Subject Headings (MeSH). A estratégia completa encontra-se no material suplementar (Quadro $1)$.

\begin{tabular}{|l|}
\hline \multicolumn{1}{|c|}{ Quadro 1 - Estratégia de busca usada para todas as bases de dados } \\
\hline (Music OR musician OR musicians) AND (Mismatch Negavitity OR MMN OR \\
Evoked Potentials Auditory OR Long Latency) \\
\hline
\end{tabular}

Fonte: Autores (2021).

\section{Critérios de Seleção}

Como critérios de inclusão foram considerados: estudos comparativos que tinham como população músicos profissionais com experiência de no mínimo 4 anos, e não músicos, indivíduos sem nenhuma experiência musical ou tendo experiencias com a música de forma não profissional por no máximo 2 anos, ambos os grupos deveriam ser adultos com faixa etária de 18 até 55 anos de idade, sem restrição de gênero, tendo sido submetidos a avaliação de Mismatch Negativity (MMN). Como critérios de exclusão os estudos tinham indivíduos que não poderiam apresentar perdas auditivas, patologias centrais, periféricas e vestibulares e idade maior que 55 anos, também foram excluídos estudos que não utilizaram MMN como método de avaliação, estudos repetidos em bases de dados diferentes.

\section{Extração de Dados}

Os títulos e resumos foram avaliados por dois pesquisadores independentes, os pesquisadores estavam cientes dos autores e ou títulos dos periódicos. Em casos de divergências um terceiro pesquisador avaliava os artigos e tomava a decisão final. Os textos completos foram lidos na integrada, assim verificando se existia diferença entre latências e amplitudes de MMN entre músicos e não músicos sendo esse o desfecho procurado. Outras informações como título, nomes dos autores, ano de publicação, faixa etárias dos grupos, e número de sujeitos em cada grupo, foram extraídos. 


\section{Resultados}

Foram identificados inicialmente nas bases de dados 1.600 títulos relevantes, 53 títulos foram excluídos por serem repetidos, 101 resumos foram avaliados no qual 30 textos completos foram lidos na integra. 21 textos foram excluídos após leitura integral por não atenderem aos critérios de inclusão (Quadro 2). Em fim foram considerados 9 textos completos relevantes para a pesquisa (Quadro 3). O fluxograma que demostra o processo de busca e seleção dos artigos está representado abaixo (Fluxograma 1).

A publicação de 1997 (M. Tervaniemi, Ilvonen, Karma, Alho, \& Näätänen, 1997) foi identificada como a mais antiga dos artigos selecionados, sendo a mais recente publicada em 2020 (Arndt, Schlemmer, \& van der Meer, 2020) , os locais de publicações dos artigos com maior número no total 3 (Arndt et al., 2020; M. Tervaniemi et al., 2009; Mari Tervaniemi, Just, Koelsch, Widmann, \& Schröger, 2005) foram publicadas na Alemanha. Os demais artigos publicados foram 3 escritos na Finlândia (M. Tervaniemi et al., 1997; Virtala, Huotilainen, Partanen, \& Tervaniemi, 2014; Vuust, Brattico, Seppänen, Näätänen, \& Tervaniemi, 2012), 1 nos Estados Unidos (Nikjeh et al., 2009), 1 na índia (Sanju \& Kumar, 2016) e 1 na Espanha (Crespo-Bojorque, Monte-Ordoño, \& Toro, 2018).

O tamanho das amostras dos grupos de músicos e não músicos eram bem variáveis, tendo no grupo de músicos amostras com apenas 13 músicos (Mari Tervaniemi et al., 2005) até 67 músicos (Nikjeh et al., 2009), já nos grupos dos não músicos o estudo com menor número de participantes teve apenas 11 não músicos (Vuust et al., 2012), e teve como maior número 51 não músicos participantes (Arndt et al., 2020). Os participantes tinham em média de 18 anos a 55 anos de idade.

Diante dos antigos selecionados 7 apresentaram menor latência e maior amplitude para músicos representando assim uma melhor discriminação auditiva em comparação aos não músicos, em contraste com esses estudos foram encontrados 2 artigos em que músicos e não músicos não diferiram em suas latências e amplitude.

Quadro 2 - Textos completos excluídos da análise.

\begin{tabular}{|l|c|c|}
\hline \multicolumn{1}{|c|}{ Autor (Ano) } & Local & Motivo \\
\hline Zuijen et al. (2004) & Finlândia & Não abordou Latência/Amplitude MNN \\
\hline Fujioka et al. (2005) & Estados Unidos & Não abordou latência/Amplitude de MMN \\
\hline Dawson et al.(2018) & Suíça & Não abordou latência/Amplitude de MMN \\
\hline Goydke et al.(2004) & Alemanha & Estudo não comparativo \\
& Japão & Não abordou latência/Amplitude de MMN \\
\hline Yumoto et al.(2005) & Canadá & Não abordou latência/Amplitude de MMN músicos) \\
\hline Zendel et al. (2008) & & Não abordou latência/Amplitude de MMN \\
\hline
\end{tabular}


Research, Society and Development, v. 10, n. 1, e59610112170, 2021

(CC BY 4.0) | ISSN 2525-3409 | DOI: http://dx.doi.org/10.33448/rsd-v10i1.12170

\begin{tabular}{|c|c|c|}
\hline Matsuda et al. (2013) & Japão & $\begin{array}{c}\text { Estudo não comparativo } \\
\text { (Grupo apenas de não músicos) }\end{array}$ \\
\hline Lidji et al. (2010) & Canadá & $\begin{array}{c}\text { Estudo não comparativo } \\
\text { (Grupo apenas de não músicos) }\end{array}$ \\
\hline Zhang et al. (2015) & China & Não abordou latência/Amplitude de MMN \\
\hline Baumann et al. (2008) & Suíça & Não abordou latência/Amplitude de MMN \\
\hline Zhang et al. (2019) & China & $\begin{array}{c}\text { Estudo não comparativo } \\
\text { (Grupo apenas de não músicos) }\end{array}$ \\
\hline Kamiyama et al. (2010) & Japão & $\begin{array}{c}\text { Estudo não comparativo } \\
\text { (Grupo apenas de não músicos) }\end{array}$ \\
\hline Rodrigues et al. (2010) & Brasil & $\begin{array}{l}\text { Revisão de literatura, não abordou amplitude e latência } \\
\text { de MMN. }\end{array}$ \\
\hline Carcagno et al. (2011) & França & $\begin{array}{c}\text { Estudo não comparativo } \\
\text { (Grupo apenas de não músicos) }\end{array}$ \\
\hline Pei et al. (2003) & Taiwan & $\begin{array}{c}\text { Estudo não comparativo } \\
\text { (Grupo apenas de não músicos) }\end{array}$ \\
\hline Koelsch et al. (2003) & Estados Unidos & $\begin{array}{c}\text { Estudo não comparativo } \\
\text { (Grupo apenas de não músicos) }\end{array}$ \\
\hline Musacchia et al. (2008) & Estados Unidos & Não abordou latência/Amplitude de MMN \\
\hline Leino et al. (2007) & Finlândia & Grupo pesquisado, não era músicos e nem não músicos. \\
\hline Zendel et al. (2015) & Estados Unidos & Não abordou latência/Amplitude de MMN \\
\hline Ruèsseler et al. (2001) & Alemanha & Não abordou latência/Amplitude de MMN \\
\hline
\end{tabular}

Fonte: Autores (2020).

No quadro acima (Quadro 2), demostra os textos completos que foram excluídos após a leitura do texto completo, de acordo com os critérios de elegibilidade. Destes 21 textos excluídos: 11 não abordam amplitudes e latências de MMN, 8 eram estudos não comparativos, ou seja, não comparavam os grupos músicos e não músicos, 1 revisão sistemática que não abordou latências e amplitudes, e 1 estudos não tinham como grupo músicos e não músicos. 
Quadro 3 - Características dos estudos incluídos.

\begin{tabular}{|c|c|c|c|}
\hline Autor (Ano) & Local & Objetivo & Resultados de latência e amplitude. \\
\hline $\begin{array}{l}\text { Bojorque et al. } \\
\text { (2018) }\end{array}$ & Espanha & $\begin{array}{l}\text { Analisar correlatos neurais subjacentes a tais benefícios de } \\
\text { processamento para consonância e mostrar como eles } \\
\text { podem ser modulados pela experiência. }\end{array}$ & $\begin{array}{c}\text { Músicos apresentaram menor latência e } \\
\text { maior amplitude em comparação a não } \\
\text { músicos. }\end{array}$ \\
\hline $\begin{array}{l}\text { Virtala et al. } \\
\text { (2014) }\end{array}$ & Finlândia & $\begin{array}{l}\text { Analisar os efeitos da musicalidade na discriminação } \\
\text { neural e comportamental de acordes de música ocidental. }\end{array}$ & $\begin{array}{l}\text { Músicos apresentaram menor latência e } \\
\text { maior amplitude em comparação a não } \\
\text { músicos. }\end{array}$ \\
\hline $\begin{array}{l}\text { Tervaniemi et } \\
\text { al. }(2005)\end{array}$ & Alemanha & $\begin{array}{l}\text { Analisar a precisão do processamento de pitch em } \\
\text { músicos com experiência em tocar uma ampla seleção de } \\
\text { instrumentos (por exemplo, piano; instrumentos de sopro } \\
\text { e cordas). }\end{array}$ & $\begin{array}{l}\text { Músicos e não músicos não diferiram } \\
\text { entre latência e amplitude. }\end{array}$ \\
\hline $\begin{array}{l}\text { Sanju et al. } \\
\text { (2016) }\end{array}$ & Índia & $\begin{array}{c}\text { verificar as habilidades de discriminação auditiva pré- } \\
\text { atenta em músicos vocais clássicos indianos e não } \\
\text { músicos }\end{array}$ & $\begin{array}{c}\text { Músicos apresentaram menor latência e } \\
\text { maior amplitude em comparação a não } \\
\text { músicos. }\end{array}$ \\
\hline $\begin{array}{l}\text { Nikjeh et al. } \\
\text { (2009) }\end{array}$ & $\begin{array}{l}\text { Estados } \\
\text { Unidos }\end{array}$ & $\begin{array}{l}\text { Verificar se o pitch pré-atentiva discriminação e mudança } \\
\text { involuntária de atenção distinguiriam músicos de não } \\
\text { músicos e se fornecem informações sobre a influência do } \\
\text { treinamento auditivo e da experiência na função auditiva } \\
\text { central. }\end{array}$ & $\begin{array}{l}\text { Músicos comparados a não músicos } \\
\text { tinham MMN mais curto latências para } \\
\text { todos os desvios (tons harmônicos, tons } \\
\text { puros e fala), e amplitude maior. }\end{array}$ \\
\hline $\begin{array}{l}\text { Arndt et al. } \\
(2020)\end{array}$ & Alemanha & $\begin{array}{l}\text { Analisar se músicos comparados a não músicos já } \\
\text { possuem vantagens de processamento auditivo em nível } \\
\text { neural. }\end{array}$ & $\begin{array}{l}\text { Músicos e não músicos não diferiram } \\
\text { entre latência e amplitude. }\end{array}$ \\
\hline $\begin{array}{l}\text { Tervaniemi et } \\
\text { al. (1997) }\end{array}$ & Finlândia & $\begin{array}{l}\text { Verificar os pré-requisitos neurofisiológicos da } \\
\text { musicalidade na discriminação auditiva. }\end{array}$ & $\begin{array}{l}\text { Músicos apresentaram menor latência e } \\
\text { maior amplitude em comparação a não } \\
\text { músicos }\end{array}$ \\
\hline $\begin{array}{l}\text { Vuust et al. } \\
\text { (2012) }\end{array}$ & Finlândia & $\begin{array}{c}\text { Analisar o papel do estilo / gênero musical na modulação, } \\
\text { respostas neurais e comportamentais às mudanças nas } \\
\text { características musicais }\end{array}$ & $\begin{array}{l}\text { Músicos principalmente no gênero Jazz } \\
\text { apresentaram menor latência e maior } \\
\text { amplitude em comparação a não músicos }\end{array}$ \\
\hline $\begin{array}{l}\text { Tervaniem et } \\
\text { al. (2009) }\end{array}$ & Alemanha & $\begin{array}{l}\text { Verificar se os parâmetros básicos do som (frequência, } \\
\text { duração e intensidade) são codificados diferencialmente } \\
\text { entre sons de fala e música por músicos e não músicos } \\
\text { durante diferentes atenções demandas }\end{array}$ & $\begin{array}{l}\text { Músicos apresentaram menor latência e } \\
\text { maior amplitude em comparação a não } \\
\text { músicos, durante a escuta atenta, para sons } \\
\text { musicais e de fala. }\end{array}$ \\
\hline
\end{tabular}

Fonte: Autores (2020).

Os dados relatados acima (Quadro 3) mostra as características dos estudos incluídos na pesquisa. Foram 9 artigos no total, que tinham como objetos de estudos músicos e não músicos, os objetivos e locais de publicação foram diversos. 
Fluxograma 1 - Fluxograma dos artigos selecionados para a revisão sistemática de literatura.

Números de artigos nas bases de dados.

$$
(\mathrm{n}=1,600)
$$

Artigos duplicatas removidos (Títulos). $(n=53)$

Artigos avaliados (Título)

$(\mathrm{n}=1,547)$

Artigos excluídos $(\mathrm{n}=1,446)$
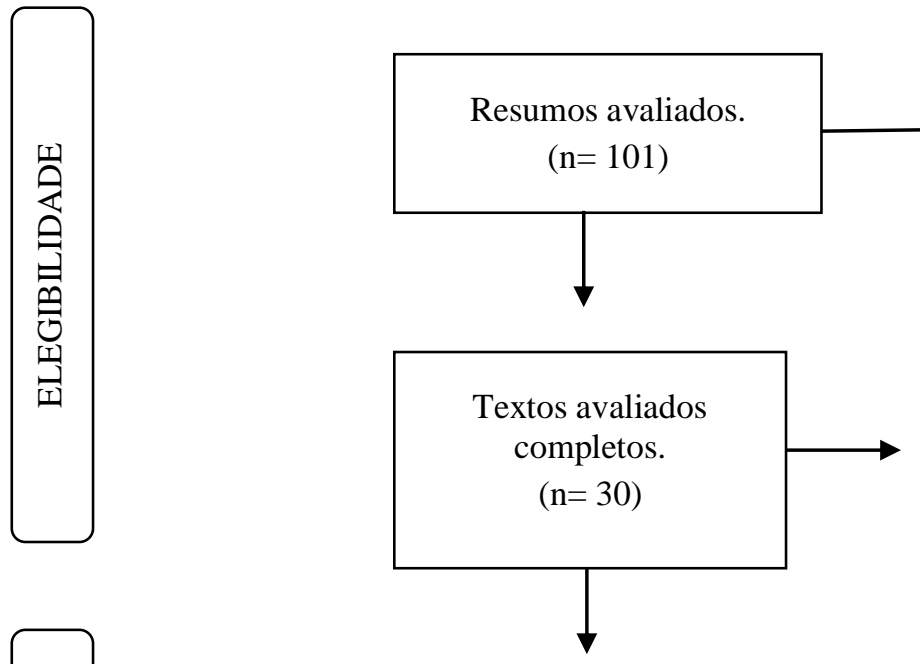

Resumos excluídos.

$$
(n=71)
$$

Textos completes excluídos.

$$
(n=21)
$$

Estudos incluídos na

$$
\text { síntese. }
$$

$(n=9)$ 


\section{Discussão}

Para estudar a discriminação auditiva pré-atentiva e armazenamento de regularidades nas características de estímulos, o potencial relacionado a eventos (ERP) Mismatch negativity (MMN) é utilizado de forma objetiva para demostrar diferenças neuronais incitadas por sons desviantes e sons padrões que geram um pico em torno de 100 a $200 \mathrm{~ms}$. A percepção auditiva encontrada nos músicos é fruto de anos de treinamento e experiencia que exige cognição e conhecimento (Sanju \& Kumar, 2016).

O estudo de (Sanju \& Kumar, 2016) relataram que as amplitudes de MMN eram mais confiáveis que suas latências, com isto foram encontrados estudos que abordavam apenas amplitudes de MNN. Este mesmo estudo revelou uma latência de início, pico de amplitude e área de pico sob a curva do MMN em músicos vocais clássicos indianos melhores em comparação a não músicos, indicando uma melhor pré-atenção nas habilidades de discriminação auditiva em músicos vocais clássicos indianos em comparação com não músicos. Resultados semelhantes foram encontrados por (Nikjeh et al., 2009) , músicos treinados e não músicos foram avaliados no objetivo de verificar se o pitch pré-atento, discriminação e mudança involuntária de atenção distinguiriam músicos de não músicos e se forneciam informações sobre a influência do treinamento auditivo e da experiência na função auditiva central, os resultados mostraram um melhor desempenho dos músicos que apresentaram menor (melhor) latência de MMN ao processamento sensorial auditivo pré-atento para modificações de frequências em tons puros, complexos e estímulos da fala, esses resultados demostram uma variedade de modulações do sistema neural auditivo promovido pela experiência dos músicos, sobre as amplitudes de MMN, o mesmo estudo mostrou que os músicos superaram não músicos apresentando maior (melhor) amplitude a tons puros, porém não havia diferenças em amplitude em tons harmônicos e estímulos de fala. Os músicos por acharem irrelevantes os estímulos foram mais lentos na descriminação dos tons, porém quando detectaram as mudanças de frequências de tons puros os músicos automaticamente discriminaram as mudanças ainda mais cedo que os não músicos sem aumento de amplitude demonstrando mais eficiência no processamento acústico.

Outro estudo de (Virtala et al., 2014) usaram sons senoidais e sons de piano ricos harmonicamente para investigar os efeitos da musicalidade na discriminação neural e comportamental de acordes de músicas ocidental, o MMN foi eliciado apenas em músicos superando assim os não músicos em acordes maiores e menores invertidos, esses resultados sugerem que os músicos possuem processamento aperfeiçoado em estímulos musicais complexos tanto comportamentalmente como neuralmente. Literaturas como (M. Tervaniemi et al., 1997), (M. Tervaniemi et al., 2009) e (Crespo-Bojorque et al., 2018) em consonância com as literaturas citadas ácima demostram que assuntos musicais eliciaram maiores amplitudes de MMN em músicos do que em não músicos.

No entanto as literaturas citadas acima até então investigaram os músicos apenas como um grupo unificado, (Vuust et al., 2012) traz um contexto diferente em que analisa o processamento cerebral pré atencioso(MMN), verificando se o estilo/gênero musical em músicos podem influenciar nas habilidades auditivas, medidas pelas respostas comportamentais e cerebrais, o estudo compara músicos e não músicos também. Levando em consideração as habilidades auditivas em geral os músicos de jazz se sobressaíram aos outros músicos estudados, apresentando amplitudes maiores de MMN, todos os outros músicos estudados superaram os não músicos, esses resultados revelam que o estilo/gênero, o tipo de treinamento e experiencias de escuta influencia as habilidades perceptivas e o processamento cerebral dos músicos.

No entanto, foram encontrados nesta pesquisa estudos que não estão de acordo com os resultados encontrados citados anteriormente (Mari Tervaniemi et al., 2005), revelou em seus resultados que músicos e não músicos não diferiram em suas amplitudes de MMN na condição de leitura, os candidatos foram expostos a sons padrões frequentes e sons raros desviantes em $0,8 \%, 2 \%$ e $4 \%$, essa não diferenciação das amplitudes foi atribuída a experiencia musical que poderia ter exercido seus efeitos apenas em processamento de nível atento, mas não no nível pré-atento. Foi encontrado resultados semelhantes no 
estudo de (Arndt et al., 2020) onde os músicos e não músicos eram expostos a padrões de sons frequentes (528 hertz) e sons desviantes raros ( $535 \mathrm{~Hz}$ e $558 \mathrm{~Hz}$ ), músicos e não músicos não diferiram significativamente em suas amplitudes de MMN , a única condição que os músicos apresentaram amplitudes maiores foi na condição de $535 \mathrm{HZ}$ com relação a não músicos, porem na condição de $558 \mathrm{HZ}$ ambos os grupos identificaram facilmente as diferença dos desvios padrão, como resultado músicos e não músicos não diferiram em suas amplitudes.

\section{Considerações Finais}

Foi possível observar que na maioria dos estudos os músicos apresentaram menor latência e maior amplitude em comparação a não músicos. Isso nos revela que existe diferença entre as latências e amplitudes em músicos e não músicos, além de demostrar melhores habilidades de discriminação auditiva e processamento sensorial pré-atenta em músicos em comparação com não músicos.

\section{Referências}

Arndt, C., Schlemmer, K., \& van der Meer, E. (2020). Same or different pitch? Effects of musical expertise, pitch difference, and auditory task on the pitch discrimination ability of musicians and non-musicians. Experimental Brain Research, 238(1), 247-258. Springer Berlin Heidelberg. https://doi.org/10.1007/s00221-019-05707-8

Crespo-Bojorque, P., Monte-Ordoño, J., \& Toro, J. M. (2018). Early neural responses underlie advantages for consonance over dissonance. Neuropsychologia, 117, 188-198. Elsevier Ltd. https://doi.org/10.1016/j.neuropsychologia.2018.06.005

Ferreira, D. A., Bueno, C. D., Costa, S. S. da, \& Sleifer, P. (2017). Aplicabilidade do Mismatch Negativity na população infantil: revisão sistemática de literatura. Audiology - Communication Research, 22(0), 1-10.

Nikjeh, D. A., Lister, J. J., \& Frisch, S. A. (2009). Preattentive cortical-evoked responses to pure tones, harmonic tones, and speech: Influence of music training. Ear and Hearing, 30(4), 432-446.

Pereira, A. S., Shitsuka, D. M., Parreira, F. J., \& Shitsuka, R. (2018). Método Qualitativo, Quantitativo ou Quali-Quanti. Metodologia da Pesquisa Científica. https://repositorio.ufsm.br/bitstream/handle/1/15824/Lic_Computacao_Metodologia-Pesquisa-Cientifica.pdf?sequence=1.

Rodrigues, A. C., Loureiro, M., \& Caramelli, P. (2013). Efeitos do treinamento musical no cérebro: aspectos neurais e cognitivos. Neuropsicologia Latinoamericana, 5(4), 15-31.

Sanju, H. K., \& Kumar, P. (2016). Pre-attentive auditory discrimination skill in Indian classical vocal musicians and non-musicians. Journal of Otology, 11(3), 102-110. Elsevier Ltd. http://dx.doi.org/10.1016/j.joto.2016.06.002

Tervaniemi, M., Ilvonen, T., Karma, K., Alho, K., \& Näätänen, R. (1997). The musical brain: Brain waves reveal the neurophysiological basis of musicality in human subjects. Neuroscience Letters, 226(1), 1-4.

Tervaniemi, M., Kruck, S., De Baene, W., Schröger, E., Alter, K., \& Friederici, A. D. (2009). Top-down modulation of auditory processing: Effects of sound context, musical expertise and attentional focus. European Journal of Neuroscience, 30(8), 1636-1642.

Tervaniemi, Mari, Just, V., Koelsch, S., Widmann, A., \& Schröger, E. (2005). Pitch discrimination accuracy in musicians vs nonmusicians: An event-related potential and behavioral study. Experimental Brain Research, 161(1), 1-10.

Virtala, P., Huotilainen, M., Partanen, E., \& Tervaniemi, M. (2014). Musicianship facilitates the processing of Western music chords-An ERP and behavioral study. Neuropsychologia, 61(1), 247-258. Elsevier. http://dx.doi.org/10.1016/j.neuropsychologia.2014.06.028

Vuust, P., Brattico, E., Seppänen, M., Näätänen, R., \& Tervaniemi, M. (2012). The sound of music: Differentiating musicians using a fast, musical multifeature mismatch negativity paradigm. Neuropsychologia, 50(7), 1432-1443. Elsevier Ltd. http://dx.doi.org/10.1016/j.neuropsychologia.2012.02.028 